\title{
Monogenoidea (Dactylogyridae) em tilápias-do-nilo cultivadas sob diferentes densidades de estocagem em tanques-rede
}

[Monogenoidea (Dactylogyridae) in Nile tilapia cultured under different stocking densities in cages]

\author{
N.G. Marengoni ${ }^{1,3}$, R.S. Santos ${ }^{2}$, A.C. Gonçalves Júnior ${ }^{1,3}$, D.M. Gino ${ }^{2}$, D.C.P. Zerbinatti ${ }^{2}$, F.S. Lima ${ }^{2}$ \\ ${ }^{1}$ Centro de Ciências Agrárias - UNIOESTE \\ Rua Pernambuco, 1777 \\ 85960-000 - Marechal Cândido Rondon, PR \\ ${ }^{2}$ Zootecnista autônomo \\ ${ }^{3}$ Grupo de Estudos em Solos e Meio Ambiente - Marechal Cândido Rondon, PR
}

\begin{abstract}
RESUMO
Avaliaram-se a prevalência e a intensidade de infecção por Monogenoidea em tilápias-do-nilo (Oreochromis niloticus) cultivadas sob diferentes densidades de estocagem em tanques-rede de $4 \mathrm{~m}^{3}$. O delineamento experimental foi inteiramente ao acaso, com quatro tratamentos - densidades (D) de $\mathrm{D}_{1}=250, \mathrm{D}_{2}=300, \mathrm{D}_{3}=350$ e $\mathrm{D}_{4}=400$ peixes $/ \mathrm{m}^{3}$, três repetições por tratamento, utilizando-se 360 peixes capturados e analisados no período de fevereiro a junho de 2003. Os parâmetros físico-químicos da água foram mensalmente monitorados. Observaram-se prevalências de 52,8; 62,5;69,4;83,3; 58,3\% e intensidade média parasitária de 112,8; 65,7; 274,0; 97,8; 100,2 de Dactylogyrus sp. (Monogenoidea: Dactylogyridae). Não houve relação da prevalência e intensidade de infecção com a densidade de cultivo, porém foi verificado efeito quadrático $(\mathrm{P}<0,05)$ da intensidade parasitária em função do mês. Verificaram-se maior intensidade média de infecção (274) e elevada prevalência $(83,3 \%)$ nos meses em que a temperatura e a transparência da água oscilaram abaixo dos limites recomendados para o conforto térmico e bem-estar das tilápias.
\end{abstract}

Palavras-chave: peixe, Oreochromis niloticus, infecção parasitária, Dactylogyrus sp., prevalência

\begin{abstract}
The prevalence and infection intensity of Monogenoidea in Nile tilapia (Oreochromis niloticus) cultivated under different stocking densities in $4 \mathrm{~m}^{3}$ cages were evaluated. The experiment was designed in sample random sampling with four treatments - densities (D) of $D_{1}=250, D_{2}=300, D_{3}=350$, and $D_{4}=400 \mathrm{fish} / \mathrm{m}^{3}$ and three repetitions, using 360 fish captured and analyzed during the period from February to June 2003. Physiochemical parameters of the water were monthly monitored. Prevalences of 52.8; 62.5; 69.4; 83.3; and 58.3\% and parasitic mean intensities of 112.8; 65.7; 274.0; 97.8; and 100.2 Dactylogyrus sp. (Monogenoidea: Dactylogyridae) were observed. No relationship between the prevalence and infection intensity with the cultivation density was found. However, quadratic effect was verified $(P<0.05)$ related to the parasitic intensity in function of the month. High mean intensity of infection (274) and high prevalence (83.3\%) were verified in the months in that the temperature and the transparency of the water oscillated below the recommended limits for the thermal comfort and well-being of tilapia.
\end{abstract}

Keywords: fish, Oreochromis niloticus, parasitic infection, Dactylogyrus sp., prevalence

\section{INTRODUÇÃO}

Os microrganismos vivem em equilíbrio no ambiente, no corpo e até nas vísceras dos peixes
(Roberts e Bullock, 1980). Assim, qualquer fator que tenha efeito direto na saúde do peixe e diminua sua resistência imunológica contribui para que os agentes patogênicos proliferem,

Recebido em 8 de abril de 2008

Aceito em 10 de março de 2009

E-mail: nmarengoni@hotmail.com 
levando o hospedeiro à morte e causando consideráveis perdas econômicas na aquicultura (Moraes e Martins, 2004).

Protozoários como Trichodina compacta e Ichthyophthirius multifiliis e os Monogenoidea, Dactylogyrus e Gyrodactylus, destacam-se como sendo os ectoparasitos de maior importância em tilápia (Martins, 1998; Martins et al., 2002; Zanolo e Yamamura, 2006). Os Monogenoidea estão incluídos entre os parasitos mais significativos para a piscicultura, uma vez que podem provocar taxa de mortalidade bastante elevada (Pavanelli et al., 2008).

Recentes estudos de infecções parasitárias em peixes cultivados no Estado de São Paulo diagnosticaram os monogenoideos como os mais importantes parasitos, com ocorrência de 72,9 e 78,9\%, respectivamente, para os anos de 1999 e 2000 (Martins et al., 2002). No Estado de Santa Catarina, Azevedo et al. (2006) encontraram trichodinídeos, Monogenoidea e Lamproglena sp., como a fauna parasitária mais importante em pele e guelras de tilápias cultivadas em diferentes regiões.

O número de parasitos aumenta em ambientes confinados e sua reprodução depende, em parte, da qualidade de água e da densidade de estocagem. Os Monogenoidea são considerados agentes patogênicos estressantes e geralmente apresentam alta especificidade parasitária. A eutrofização do ambiente aquático, a concentração de oxigênio dissolvido abaixo do ideal e a alta densidade de estocagem são causadores de estresse aos peixes, predispondoos às infecções (Ostrensky e Boeger, 1998; Ceccarelli et al., 2000; Azevedo et al., 2006).

Poucos são os trabalhos relacionados à epidemiologia e à patogenia de ectoparasitos em criações de tilápia-do-nilo em tanques-rede no Brasil. Ranzani-Paiva et al. (1998) analisaram a ocorrência de ectoparasitos em exemplares de tilápia-do-nilo na represa de Guarapiranga, SP, e encontraram Trichodina sp. (7,7\%), monogenoideos $(7,7 \%)$ e I. multifiliis $(1,5 \%)$. Marengoni e Santos (2004), ao estudarem a fauna ectoparasitária da tilápia-do-nilo cultivada no reservatório da Usina Hidrelétrica de Rosana, SP, verificaram maior prevalência por Monogenoidea (68,4\%) seguido por Trichodina sp. (53,1\%), Lernaea sp. (7,1\%), Chilodonella sp. (4,1\%) e Epistylus sp. (4,1\%). Zanolo (2004) também encontrou alta prevalência (90 a $100 \%$ ) por monogenoideos nos seis meses de cultivo de tilápia-do-nilo à densidade de 250 peixes $/ \mathrm{m}^{3}$ na represa de Capivara, município de Sertaneja, Paraná.

A qualidade de água do ambiente onde estão os tanques-rede tem influência direta sobre a produtividade e a sanidade dos peixes (Kubitza, 2003). Considerando que é inviável técnica e economicamente corrigir a qualidade dos grandes corpos de água, faz-se necessário um estudo do histórico do local de produção bem como seu monitoramento ao longo do cultivo. Por outro lado, uma característica do sistema de produção de peixes em tanques-rede é a renovação contínua de água, que, apesar das altas densidades de estocagem, evita o acúmulo de matéria orgânica e o déficit na concentração de oxigênio dissolvido (Zanolo e Yamamura, 2006).

Os incidentes decorrentes de mortes de peixes por parasitas e outros fatores adjuntos são causadores de grandes prejuízos para os produtores (Ostrensky e Boeger, 1998). Na produção de tilápia em tanques-rede, os peixes podem estar condicionados a fatores estressantes, principalmente aos relacionados às altas densidades de estocagem. Dessa forma, este trabalho teve como objetivo avaliar o efeito da densidade de estocagem na ocorrência de Monogenoidea em tilápia-do-nilo (Oreochromis niloticus) cultivada em tanques-rede de baixo volume instalados em águas públicas da União.

\section{MATERIAL E MÉTODOS}

O estudo foi realizado no reservatório da Usina Hidrelétrica de Rosana, no município de Euclides da Cunha Paulista, SP, no Laboratório de Microscopia da Estação de Piscicultura da Universidade do Oeste Paulista, em Presidente Prudente, SP, e no Laboratório de Zoologia e Parasitologia da Universidade Estadual do Oeste do Paraná, em Marechal Cândido Rondon, PR.

Tilápias-do-nilo, Oreochromis niloticus Linnaeus, 1757 (Cichlidae), com peso médio inicial de $80 \mathrm{~g}$, foram distribuídas em quatro tratamentos - densidades de estocagem de 250 , 300,350 e 400 peixes $/ \mathrm{m}^{3}$-, e três repetições cada, utilizando-se de 12 tanques rede, malha $3 / 4$ de arame galvanizado revestido, com dimensões 
de 2,0 $02,0 \times 1,3 \mathrm{~m}$ de comprimento, largura e altura, respectivamente, totalizando uma área de cultivo de $4,0 \mathrm{~m}^{3}$ para cada tanque, cobertos com o mesmo material para evitar a fuga e a predação dos peixes.

Os peixes foram alimentados em comedouros apropriados e instalados nos tanques-rede, utilizando-se o sistema de alimentação manual com ração comercial extrusada contendo $32 \%$ de proteína bruta. A taxa de arraçoamento foi de 3 a $2 \%$ em relação à biomassa do tanque, ajustada de acordo com as biometrias quinzenais e a temperatura da água. A frequência de arraçoamento utilizada foi de três vezes/dia, sempre respeitando as condições da qualidade de água.

A análise parasitológica e a quantificação de parasitos seguiram as recomendações de Ghiraldelli et al. (2006b). A prevalência e a intensidade de infecção foram calculadas de acordo com Bush et al. (1997), e a identificação baseou-se em Kritsky et al. (1991) e Thatcher (1991). Os dados biométricos de peso (g) e comprimento total $(\mathrm{cm})$ dos peixes foram utilizados para determinar o fator relativo de condição $(\mathrm{Kn})$ de acordo com Carneiro et al. (1999).

As variações observadas durante o experimento para os parâmetros aquáticos, como oxigênio dissolvido $(3,21$ a $6,45 \mathrm{mg} / \mathrm{L}), \mathrm{pH}(5,59$ a 6,87$) \mathrm{e}$ condutividade $\left(20,54\right.$ a $\left.82,99 \mu \mathrm{S} / \mathrm{cm}^{2}\right)$ estavam adequadas aos limites de conforto da espécie e não tiveram influência significativa no desempenho dos peixes (Kubitza e Kubitza, 2000; Kubitza, 2003). A temperatura da água do reservatório variou de 19 a $28,64^{\circ} \mathrm{C}$, com valores médios de 24,53, 20,50 e $23,0^{\circ} \mathrm{C}$, respectivamente, em abril, maio e julho, oscilando abaixo dos limites adequados para o conforto térmico da espécie tropical estudada, conforme sugerido por Galli e Torloni, (1999) e Ono e Kubitza (2003). A transparência da água, monitorada utilizando-se o do disco de Secchi, variou de 76,50 a $113,17 \mathrm{~cm}$, caracterizando o ambiente como eutrofizado e favorável à propagação dos monogenoideos (Tab. 1).

Tabela 1. Parâmetros aquáticos (média \pm desvio-padrão) no reservatório de Rosana, São Paulo, Brasil, entre fevereiro a julho de 2003, durante o cultivo de tilápia-do-nilo em tanques-rede

\begin{tabular}{lrrrrc}
\multicolumn{1}{c}{ Parâmetro } & Fevereiro & Março & Abril & Maio & Junho \\
& & & & & \\
\hline pH & $6,23 \pm 0,37$ & $6,04 \pm 0,56$ & $6,11 \pm 0,85$ & $6,79 \pm 0,26$ & $6,48 \pm 0,38$ \\
Oxigênio (mg/L) & $5,08 \pm 1,73$ & $4,04 \pm 1,46$ & $5,16 \pm 2,34$ & $6,10 \pm 1,50$ & $5,10 \pm 1,27$ \\
& & & & & \\
$\begin{array}{l}\text { Condutividade } \\
(\mu \mathrm{S} / \mathrm{cm})\end{array}$ & $61,39 \pm 3,98$ & $52,15 \pm 4,33$ & $43,50 \pm 5,28$ & $63,89 \pm 4,69$ & $38,60 \pm 5,74$ \\
$\begin{array}{l}\text { Temperatura }\left({ }^{\circ} \mathrm{C}\right) \\
\text { Transparência }\end{array}$ & $28,05 \pm 1,84$ & $27,20 \pm 2,45$ & $24,53 \pm 1,93$ & $20,50 \pm 2,37$ & $23,00 \pm 1,61$ \\
& $108,22 \pm 2,05$ & $96,78 \pm 1,20$ & $97,85 \pm 2,19$ & $103,01 \pm 2,45$ & $110,00 \pm 3,05$ \\
$(\mathrm{~cm})$ & & & & & \\
\hline
\end{tabular}

Os valores médios observados foram submetidos às análises de variância e regressão e ao teste $\mathrm{t}$ ou Tukey para comparação de médias, relacionando a intensidade média parasitária com o mês de cultivo e a densidade estudada, utilizando-se o programa computacional SAEG (Sistema ..., 1999).

\section{RESULTADOS E DISCUSSÃO}

Prevalências de 52,8; 62,5;69,4;83,3; 58,3\% e intensidade média parasitária de 112,$8 ; 65,7 ; 274,0$;
97,8; 100,2 de Dactylogyrus sp. (Monogenoidea: Dactylogyridae), respectivamente, para os meses de fevereiro, março, abril, maio e junho de 2003, são apresentadas na Tab. 2. Estes valores, apesar de serem considerados altos, não resultaram em nenhum transtorno aparente no cultivo dos peixes, pois o fator de condição variou de 1,67 a 2,17, sendo considerado bom (Gichuri e Omondi, 2003). Zanolo (2004) também encontrou alta prevalência de monogenoideos (90 a 100\%) durante os seis meses de cultivo de tilápia-do-nilo à densidade de 
250 peixes $/ \mathrm{m}^{3}$ na represa de Capivara, município de Sertaneja, PR. Pavanelli et al. (2008) concluíram que o prejuízo determinado nos peixes está relacionado com a espécie do parasito, com o local de ocorrência, intensidade da infestação e com o tipo de alimentação. A maioria dos hospedeiros alimenta-se de muco e células epiteliais, porém alguns podem se alimentar de sangue. Neste trabalho, os organismos monogenoideos foram encontrados alojados nos filamentos branquiais das tilápias e, após a identificação de um total de 517 parasitos, todos apresentaram as características de Dactylogyrus sp. (Monogenoidea: Dactylogyridae).

Tabela 2. Valores (média \pm desvio-padrão) do peso, comprimento, número de peixes infectados/ examinados, prevalência e intensidade média e variação de infecção por monogenoideos em tilápia-donilo cultivada no reservatório de Rosana, SP, no período de fevereiro a junho de 2003

\begin{tabular}{lccccc}
\hline Variável & Fevereiro & Março & Abril & Maio & Junho \\
\hline Peso (g) & $80,45 \pm 12,53$ & $183,78 \pm 21,3$ & $278,91 \pm 31,9$ & $401,86 \pm 34,9$ & $466,72 \pm 66,7$ \\
& & 9 & 0 & 8 & 7 \\
Comprimento total (cm) & $15,11 \pm 1,25$ & $20,17 \pm 2,88$ & $23,67 \pm 2,63$ & $26,10 \pm 3,38$ & $30,29 \pm 3,75$ \\
& & & & & \\
Fator de condição (Kn) & $2,10 \pm 0,13$ & $2,02 \pm 0,20$ & $1,99 \pm 0,29$ & $2,17 \pm 0,22$ & $1,67 \pm 0,49$ \\
& & & & & \\
Peixes infectados/examinados & $38 / 72$ & $45 / 72$ & $50 / 72$ & $60 / 72$ & $42 / 72$ \\
Prevalência (\%) & 52,8 & 62,5 & 69,4 & 83,3 & 58,3 \\
Intensidade média & $112,8 \mathrm{~b}$ & $65,7 \mathrm{~b}$ & $274,0 \mathrm{a}$ & $97,8 \mathrm{~b}$ & $100,2 \mathrm{~b}$ \\
& $(29-138)$ & $(29-138)$ & $(67-354)$ & $(11-251)$ & $(14-393)$
\end{tabular}

Valores seguidos por letras distintas na linha diferem entre si pelo teste de Tukey $(\mathrm{P}<0,05)$.

A relação entre peso e comprimento total das tilápias analisadas em cada mês foi empregada para estimar o fator de condição $(\mathrm{Kn})$ que variou entre 1,59 a 2,24 e 1,60 a 2,45 , respectivamente, para os peixes não infectados e parasitados. $\mathrm{O}$ fator relativo de condição das tilápias não foi influenciado $(\mathrm{P}<0,05)$ pelo mês (Tab. 2). O Kn já foi empregado para caracterizar o estado geral, tanto para peixes em seu habitat como em piscicultura (Braga, 1997), parasitados (TavaresDias et al., 1999) ou sadios (Tavares-Dias et al., 2000). O monitoramento dessas variáveis é importante para indicar distúrbios metabólicos que podem ocorrer durante os processos patológicos e/ou nutricionais (Lourenço et al., 2002) e poderiam também ser utilizados como informações complementares aos dados biométricos usuais nas atividades aquícolas em tanque-rede.

Não houve diferença significativa da prevalência parasitária segundo o mês ou a densidade de estocagem (Fig. 1). A intensidade de monogenoideos nas tilápias não sofreu a influência $(\mathrm{P}>0,05)$ da densidade de estocagem, porém foi verificado efeito quadrático $(\mathrm{P}<0,05)$ da intensidade de infecção em função do mês, expresso pela equação de regressão: $\mathrm{y}=$ $14,4121+122,9930 \mathrm{x}-20,3929 \mathrm{x}^{2}\left(\mathrm{R}^{2}=0,0490\right)$. A maior $(\mathrm{P}<0,05)$ intensidade foi observada no mês de abril (Tab. 2 e Fig. 2), valores acima dos encontrados por Ghiraldelli et al. (2006a) e Azevedo et al. (2006) em tilápias cultivadas em Santa Catarina. Apesar da elevada prevalência $(>80 \%)$ no mês de maio, para todas as densidades de estocagem estudadas, não foi verificada $(\mathrm{P}>0,05)$ relação entre densidade $\mathrm{e}$ ocorrência de parasitos, pois, para cada densidade, houve períodos de maior e menor infestação, diferente do encontrado por Nomura et al. (2002), que estudaram a intensidade parasitária por monogenoideos em tambacu (Piaractus mesopotamicus macho x Colossoma macropomum fêmea) e concluíram que a alta densidade de estocagem interfere na carga parasitária dos indivíduos estudados.

Baixas infestações por parasitos podem também ser responsáveis por mortalidade, desde que haja queda na qualidade de água e no oxigênio dissolvido. Essas infestações, que nitidamente incomodam os peixes, podem resultar em 
infecções secundárias por bactérias e fungos, que no Brasil são favorecidas pelo clima tropical. Dessa forma, não é descartada a hipótese de mortalidade em peixes adultos quando as condições aquáticas são inadequadas, aspecto já ressaltado por Ghiraldelli et al. (2006a) e Zanolo e Yamamura (2006), ao mostrarem a necessidade de cuidados extremos com a água, além das boas práticas de manejo que respeite a capacidade de suporte dos sistemas de produção (Ono e Kubitza, 2003).

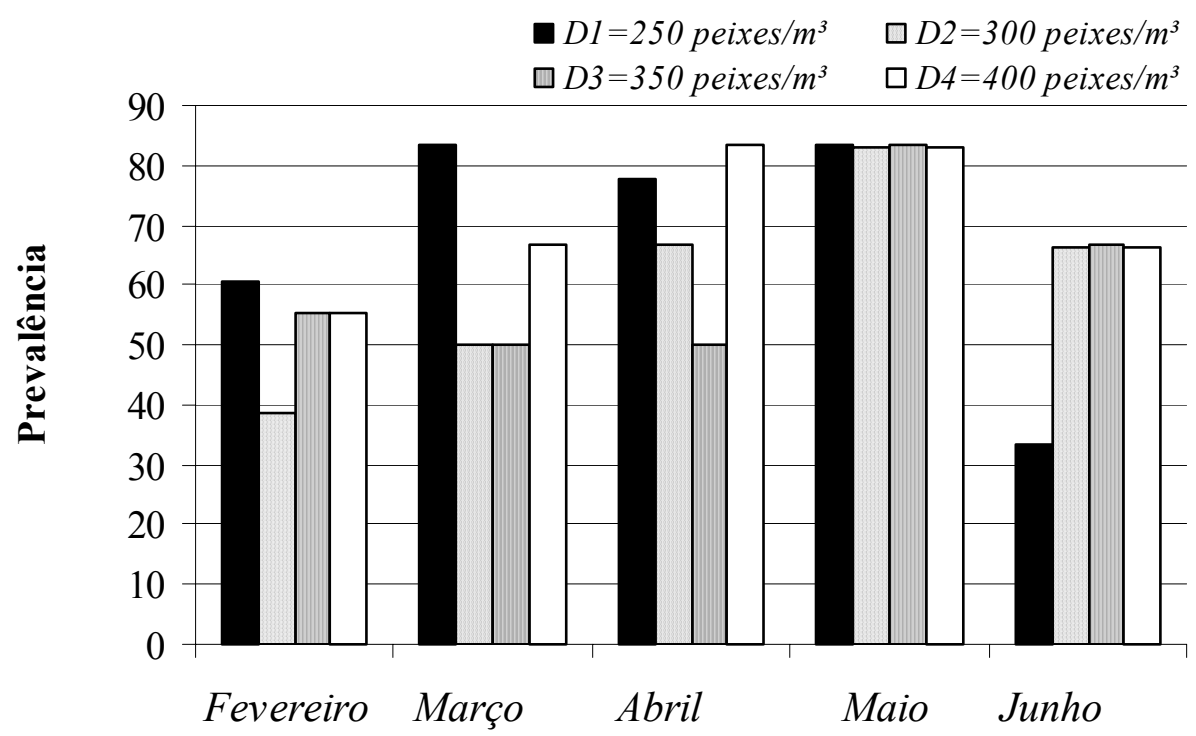

Figura 1. Prevalência mensal de Monogenoidea durante o cultivo de tilápias-do-nilo estocadas em tanques-rede sob diferentes densidades.

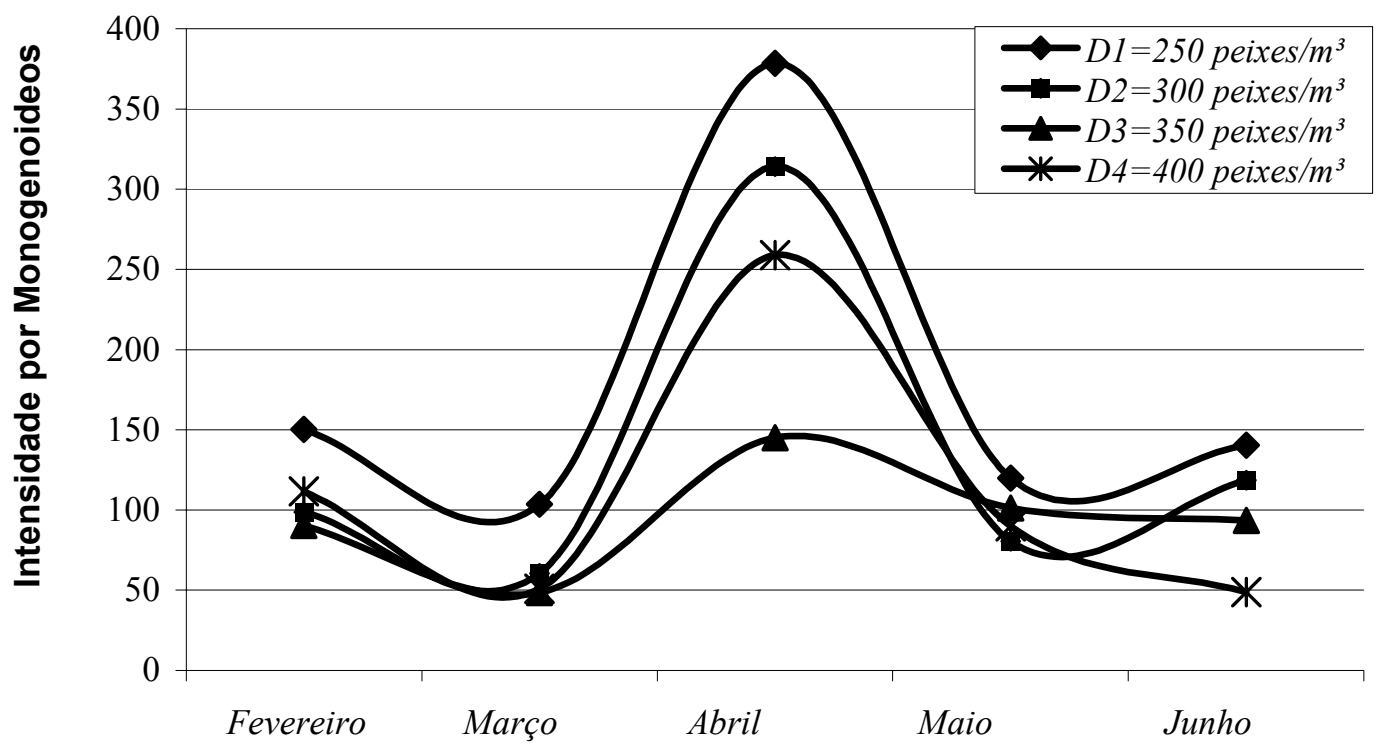

Figura 2. Intensidade de infecção e mês de estudo do parasito dactilogirídeos em brânquias de tilápia-donilo sob diferentes densidades de estocagem em tanques-rede, no reservatório de Rosana. 
Buchmann e Bresciani (1997) mostraram haver maior acometimento em truta arco-íris (Oncorhynchus mykiss) por organismos monogenoideos nos meses de janeiro, março, maio e novembro. Além disso, verificaram que esses patógenos têm relação com o tamanho dos peixes e a temperatura da água, pois quanto maior o tamanho e mais baixa a temperatura da água, maior é a incidência parasitária.

Para Kubitza e Kubitza (2000) e Marengoni et al. (2001), a susceptibilidade das tilápias às parasitoses e a outras doenças depende de diversos fatores, tais como: espécie ou linhagem, qualidade da água e carga orgânica nas unidades de produção, estado nutricional dos peixes e, principalmente, condições de temperatura da água. Nas condições estudadas, as tilápias mostraram certa rusticidade e especificidade parasitária, pois estavam submetidas ao ambiente de reservatório de usinas hidrelétricas povoados com espécies autóctones de peixes hospedeiros de muitas faunas parasitárias.

Garcia et al. (2002, 2003), ao trabalharem com peixe espada (Xiphophorus sp.), constataram que a variação na intensidade de parasitismo por monogenoideos está relacionada às variações dos parâmetros da água. Assim, em todos os tanquesrede, a renovação constante da água e a grande carga orgânica disposta podem ter favorecido o aparecimento dos parasitos. Em condições de meio ambiente eutrófico, onde os níveis de nutrientes são elevados, o oxigênio dissolvido com índices baixos e a temperatura inadequada do tanque desencadeiam os fatores que estressam os peixes. Esse estresse resulta em queda na capacidade imunológica, levando à maior susceptibilidade de os organismos adquirirem certos patógenos, comprometendo parcialmente sua sanidade e a qualidade do produto final.

\section{CONCLUSÕES}

Não se observou correlação entre densidade de estocagem de tilápia-do-nilo em tanques-rede e prevalência ou intensidade de infestação por monogenoideos. Também não houve relação entre parasitismo das brânquias das tilápias e mês de ocorrência, porém a regressão quadrática foi a que melhor ajustou o efeito do mês para intensidade de infestação. Outros fatores podem também estar associados ao parasitismo, sugerindo avaliar a sazonalidade de ocorrência e a relação entre monogenoideos-ambiente-tilápias cultivadas no reservatório da Usina Hidrelétrica de Rosana, SP.

\section{AGRADECIMENTOS}

Aos professores Dr. Maurício Laterça Martins, pelas valiosas sugestões no manuscrito e Dr. Rodrigo de Almeida Teixeira, pela análise estatística. À Pró-Reitoria de Pesquisa e Pósgraduação da UNOESTE, pelo suporte financeiro para a execução deste trabalho.

\section{REFERÊNCIAS BIBLIOGRÁFICAS}

AZEVEDO, T.M.P.; MARTINS, M.L.; BOZZO, F.R. et al. Hematological and gill responses in parasitized tilapia from Valley of Tijucas River, SC, Brazil. Sci. Agric., v.63, p.115-120, 2006.

BRAGA, F.M.S. Análise da equação alométrica na relação peso e comprimento e o fator de condição em Plagioscion squamosissinus (Teleostei, Scianidae). Rev. Bras. Biol., v.53, p.417-425, 1997.

BUCHMANN, K.; BRESCIANI, J. Parasitic infections in pond-reared rainbow trout Oncorhynchus mykiss in Denmark. Dis. Aquat. Org., v.28, p.125-138, 1997.

BUSH, A.O.; LAFFERTY, K.D.; LOTZ, J.M. et al. Parasitology meets ecology on its own terms: Margolis et al. Revisited. J. Parasitol., v.83, p.575-583, 1997.

CARNEIRO, P.C.F.; CYRINO, J.E.P.; CASTAGNOLLI, N. Produção da tilápia vermelha da Flórida em tanques-rede. Sci. Agric., v.56, p.673-679. 1999.

CECCARELLI, P.S.; SENHORINI, J.A.; VOLPATO, G.L. Dicas de piscicultura: perguntas \& respostas. Botucatu: Santana, 2000. $247 \mathrm{p}$.

GALLI, L.F.; TORLONI, C.E.C. Criação de peixes. 3.ed. São Paulo: Nobel, 1999. 119p.

GARCIA, F.G.; FUJIMOTO, R.Y.; MARTINS, M.L. et al. Estudo de parasitose por monogenoides em peixe espada (Xiphophorus sp.) em uma piscicultura de peixes ornamentais do município de Araraquara, SP. In: SIMPÓSIO BRASILEIRO DE AQUICULTURA, 12., 2002, Goiânia. Anais... Goiânia: ABRAq/UFG, 2002. CD-ROM. (Resumo). 
GARCIA, F.G.; FUJIMOTO, R.Y.; MARTINS, M.L. et al. Parasitismo de Xiphophorus spp. por Urocleidoides sp. e sua relação com os parâmetros hídricos. Bol. Inst. Pesca, v.29, p.123-131, 2003.

GICHURI, W.M; OMONDI, J.G. Relative condition factors $\mathrm{Kn}$ for Oreochromis niloticus Cichlidae and Clarias gariepinus Clariidae in small managed ponds in Kenya. In: WORLD AQUACULTURE, 2003, Salvador. Abstracts... Salvador: INVE, 2003. v.2, p.311. (Resumo).

GHIRALDELLI, L.; MARTINS, M.L.; JERÔNIMO, T.G. et al. Ectoparasites communites from Oreochromis niloticus cultivated in the State of Santa Catarina, Brazil. J. Fish. Aquat. Sci., v.1, p.181-190, 2006a.

GHIRALDELLI, L.; MARTINS, M.L.; YAMASHITA, M.M. et al. Ectoparasites influence on the hematological parameters of Nile tilapia and carp cultivated in the State of Santa Catarina, South Brazil. J. Fish. Aquat. Sci., v.1, p.270-276, 2006b.

KRITSKY, D.C.; VIDAL MARTÍNEZ, V.M.; RODRIGUES-CANUL, R. Neotropical Monogenoidea. 19. Dactylogyridae of cichlids (Perciformes) from the Yucatán Península, with descriptions of three new species of Sciadicleithrum Kritsky, Thatcher and Boeger, 1989. J. Helminthol. Soc. Washington, v.61, p.26-33, 1991.

KUBITZA, F. Qualidade da água no cultivo de peixes e camarões. Jundiaí: F. Kubitza, 2003. $229 \mathrm{p}$.

KUBITZA, L.M.; KUBITZA, F. Principais parasitoses e doenças em tilápia. Panor. Aquic., v.10, p.39-53, 2000.

LOURENÇO, J.N.P.; VARELLA, A.M.B.; MALTA, J.C.O. et al. Índice hepatossomático de tambaquis Colossoma macropomum (Cuvier, 1818) cultivados em tanques-rede. In: SIMPÓSIO BRASILEIRO DE AQUICULTURA, 12., 2002, Goiânia. Anais... Goiânia: ABRAq/UFG, 2002. CD-ROM. p.280 (Resumo).

MARENGONI, N.G.; BITTENCOURT, F.; SANTOS, M.R.F. et al. Prevalência e intensidade média de ectoparasitas em alevinos sexualmente revertidos em três linhagens de tilápia (Oreochromis sp.) em hapas. In: CONGRESSO BRASILEIRO DE
ENGENHARIA DE PESCA, 12., 2001, Foz do Iguaçu. Anais... Foz do Iguaçu: CONBEA, 2001, CD-ROM. (Resumo).

MARENGONI, N.G.; SANTOS, R.S. Monitoramento da fauna ectoparasitária da tilápia do Nilo, Orechromis niloticus (Trewavas, 1983), cultivada em tanques-rede. In: SIMPÓSIO BRASILEIRO DE AQUICULTURA, 13., 2004, Fortaleza. Anais... Fortaleza: ABRAq, 2004. p.260. (Resumo).

MARTINS, M.L. Doenças infecciosas e parasitárias de peixes. 2.ed. Jaboticabal: FUNEP/UNESP, 1998. 66p. (Boletim Técnico).

MARTINS, M.L.; ONAKA, E.M.; MORAES, F.R. et al. Recent studies on parasitic infections of freshwater cultivated fish in the state of São Paulo, Brazil. Acta Sci., v.24, p.981-985, 2002.

MORAES, F.R; MARTINS, M.L. Condições predisponentes e principais enfermidades de teleósteos em piscicultura intensiva, In: CYRINO, J.E.P.; URBINATI, E.C.; FRACALOSSI, D.M. et al. (Eds). Tópicos especiais em piscicultura de água tropical intensiva. São Paulo: TecArt, 2004. p.343-383.

NOMURA, D.T.; FUJIMOTO, R.Y.; MARTINS, M.L. et al. Avaliação da intensidade parasitária por monogenéticos e da resposta fisiológica em tambacu (Piaractus mesopotamicus x Colossoma macropomum) submetido ao estresse de captura e densidade. In: SIMPÓSIO BRASILEIRO DE AQUICULTURA, 12., 2002, Goiânia. Anais... Goiânia: ABRAq/UFG, 2002. p.294. (Resumo).

ONO, E.A.; KUBITZA, F. Cultivo de peixes em tanques-rede. 3.ed. Jundiaí: E. Ono, 2003. 112p.

OSTRENSKY, A.; BOEGER, W. Piscicultura: fundamentos e técnicas de manejo. Guaíba: Agropecuária, 1998. 211p.

PAVANELLI, G.C.; EIRAS, J.C.; TAKEMOTO, R.M. Doenças de peixes: profilaxia, diagnóstico e tratamento. 3.ed. Maringá: EDUEM, 2008. 338p.

RANZANI-PAIVA, M.J.T.; FELIZARDO, N.N.; EIRAS, A.C. et al. Análise parasitológica de brânquias e pele de tilápia-do-nilo Oreochromis niloticus Linnaeus, 1757 criados em tanque-rede na represa Guarapiranga, SP. In: ENCONTRO BRASILEIRO DE PATOLOGISTAS DE ORGANISMOS AQUÁTICOS, 5., 1998. 
Maringá. Anais... Maringá: ABRAPOA/UEM, 1998. p.140. (Resumo).

ROBERTS, R.J.; BULLOCK, A.M. The skin surface ecosystem of teleost fishes. Proc. R. Soc., v.79B, p.87-91, 1980.

SISTEMA de análises estatísticas e genéticas SAEG . Viçosa: UFV, 1999. 59p.

TAVARES-DIAS, M.; MORAES, F.R.; MARTINS, M.L. Relação hepatossomática e esplenossomática em peixes teleósteos de cultivo intensivo. Rev. Bras. Zool., v.17, p.273-281, 2000.

TAVARES-DIAS, M.; SCHALCH, S.H.C.; MARTINS, M.L. et al. Hematologia de teleósteos brasileiros com infecção parasitária. I. Variáveis do Leporinus macrocephalus
Garavello e Britski, 1998 (Anostomidae) e Piaractus mesopotamicus Holmberg, 1887 (Characidae). Acta Sci., v.21, p.337-342, 1999.

THATCHER, V.E. Amazon fish parasites. Amazoniana, v.11, p.263-572, 1991.

ZANOLO, R. Influência do parasitismo branquial por monogenoideos no desenvolvimento de tilápias-do-nilo (Oreochromis niloticus) Linnaeus, 1757 criadas em sistemas de tanques-rede na represa de Capivara, PR. 2004. Dissertação (Mestrado) Universidade Estadual de Londrina, Londrina.

ZANOLO, R.; YAMAMURA, M.H. Parasitas em tilápias do Nilo criadas em sistema de tanques-rede. Semina: Cienc. Agrar., v.27, p.281-288, 2006. 